\title{
Quand la didactique du français interroge les théories linguistiques de référence sur la reformulation orale : l'atelier de production d'écrits au Cours Préparatoire
}

\author{
Hélène Castany-Owhadi \\ Laboratoire LIRDEF, EA 3749, 2 place Marcel Godechot BP 415234092 Montpellier Cedex 5, \\ France
}

\begin{abstract}
Résumé. La présente contribution prend appui sur une recherche qui étudie les effets des reformulations orales sur les apprentissages de l'écrit des élèves en contexte d'atelier de production d'écrits au Cours Préparatoire, les données provenant de la recherche Lire et Écrire au CP (IFÉ). Nous tentons de montrer les limites de l'emprunt des théories linguistiques de référence pour l'étude de la reformulation en contexte didactique, l'enseignant répétant souvent des unités linguistiques inférieures au mot en utilisant le corps et la voix afin d'aider l'élève à produire de l'écrit. Cette prise en compte nous a amenée à adopter une conception extensive de la reformulation et à concevoir une typologie des reformulations autour de l'écrit à produire ou produit, autrement dit des reformulations de l'« écrit dans l'oral ». Nous prenons en considération la lettre, le phonème, la syllabe ainsi que la multimodalité car elles sont au service de l'accompagnement didactique mais aussi l'« écrit dans l'oral » distancé que l'on peut considérer comme la trace d'une attitude réflexive sur la langue. Nous mettons ainsi en évidence des indicateurs d'expertise langagière: les reformulations de l'« écrit dans l'oral » avec modalités gestuelle/verbale du maitre ainsi que sa capacité à susciter des reformulations avec de l'« écrit dans l'oral » distancé du côté des élèves.
\end{abstract}

\begin{abstract}
When the Teaching of French Questions the Main Linguistic Theories of Reformulation : First Grade Writing Workshop. This paper is based on a research that analyses the effects of reformulations on learning to write in the context of first grade writing workshops. It provides evidence of the need to take into account linguistic units inferior to the word and teachers' body and voice to better reflect the didactic functions of reformulations in the context of first grade writing workshops. As teachers often repeat letters, phonemes and syllables in order to help students to write, we take into consideration these linguistic units but also multimodality, our research data coming from the national project for first-grade Teaching to Read-and-Write policy (IFÉ) : thus, the teaching of writing questions the main linguistic theories of reformulation. In order to categorize the reformulations related to the written production or the one to be produced,
\end{abstract}


that we call « written production contained in speech » reformulations, we establish a typology which makes it possible to identify indicators of professional language expertise : « written production contained in speech » reformulations with teacher's gesture and teacher's ability to provoke students' reformulations with commented « written production contained in speech ».

La reformulation orale a été étudiée autant dans le cadre de l'analyse des conversations ordinaires (par ex. Fuchs, 1982) que dans celui des interactions didactiques (par ex. GarciaDebanc \& Volteau, 2007). La plupart des travaux sur la reformulation orale en contexte didactique (Volteau, 2009; Moussi, 2013; Roquelaure, 2014) utilise le modèle classificatoire de Gülich et Kotschi (1987) ou celui de De Gaulmyn (1987) comme outil d'analyse : ce sont des modèles conçus dans un contexte qui n'a rien à voir avec l'école puisqu'il s'agit d'une interaction entre un conseiller juridique et un locataire dans une association de défense de locataires. Ces modèles permettent-ils de rendre suffisamment compte de la dimension didactique, autrement dit des contenus et de leurs relations à l'enseignement/apprentissage (Reuter, 2007/2010) ? Sont-ils suffisants dans le cadre de notre contexte d'étude qui est celui de l'atelier de production d'écrits au CP ? La présente contribution tente de montrer que « les recherches didactiques peuvent poser (...) quelques questions aux théories linguistiques de référence » (Garcia-Debanc, 2008).

\section{1. État des recherches et questionnement}

\subsection{La reformulation orale, un objet d'étude difficile à circonscrire}

Depuis une vingtaine d'années, les travaux francophones sur la reformulation orale en contexte scolaire tentent de montrer le rôle de ce phénomène langagier dans la construction des savoirs (Brixhe \& Spécogna, 1999 ; Jaubert \& Rebière, 2001 ; Milian, 2005 ; GarciaDebanc \& Volteau, 2007 ; Marin, 2011; Moussi, 2013) mais aussi dans les processus acquisitionnels en langue première (par ex. Martinot, 2000). Ces recherches s'inscrivent aussi dans un courant de recherche plus général qui s'intéresse aux pratiques langagières en contexte scolaire (par ex. Garcia-Debanc \& Plane, 2004), autrement dit des « usages du langage socialement construits » (Bautier, 2001, p. 125) prenant en compte les dimensions sociales, culturelles, identitaires, linguistiques, langagières et cognitives dans la construction du sujet. Dans la continuité des travaux de Peytard (1993), des recherches récentes ont mis en évidence l'importance de la prise en compte de la multimodalité et de la pluri-sémioticité dans le cadre de l'étude des reformulations orales en contexte didactique (Rabatel, 2010a).

La reformulation comme terme générique englobant toute forme de retour sur un discours antérieur a suscité plus d'engouement que le terme « reprise» (Rabatel, 2017). À partir de cette première définition, on peut s'interroger sur l'empan de discours pris en compte : doiton prendre en considération le prédicat (Martinot, 2000), le mot (Gülich \& Kotschi, 1987 ; Rabatel, 2010b), la syllabe voire le phonème (Apothéloz, 2007) comme unité minimale d'analyse?

Les travaux princeps de Fuchs (1982), Gülich et Kotschi (1983, 1987) et De Gaulmyn (1987), qui accordent une place primordiale à la paraphrase et à la relation sémantique entre un énoncé-source et un énoncé reformulateur, ont grandement influencé les recherches sur la reformulation orale, avec les consensus suivants : - la répétition est un cas limite de la reformulation ; - la paraphrase, la correction et la glose peuvent aussi être considérées comme des reformulations ; - toute reformulation a un énoncé-source (ES) et un 
énoncé reformulateur (ER) ; - la reformulation peut être « immédiate » (ES et ER contigus) ou « différée » (ES et ER séparés par des énoncés intercalés).

On peut cependant constater que la signification des termes employés lorsque l'on reprend-modifie des dires antérieurs divergent selon les chercheurs comme l'attestent les exemples suivants : une relation de " parenté sémantique » est établie entre un énoncé-source et un énoncé reformulateur pour la paraphrase selon Fuchs (1982) alors que l'« invariant sémantique » est posé comme condition de jugement de paraphrase selon Gülich et Kotschi (1987); en ce qui concerne le rephrasage (Gülich \& Kotschi, 1987) et la répétition (De Gaulmyn, 1987), les mêmes mots réapparaissent à la différence que pour la répétition « il peut y avoir abrègement, expansion, réarrangement » (De Gaulmyn, 1987, p. 87).

D'autre part, Apothéloz (2007) défend l'idée qu'il faut s'affranchir de la notion d' « invariant sémantique », les « patterns de reformulation » (Apothéloz, 2005) étant des variantes formelles de répétition. Celui-ci définit d'ailleurs la reformulation comme « une répétition purement formelle d'une séquence de format quelconque » (Apothéloz, 2007, p. 157). L'auteur étudie d'ailleurs la reformulation orale en contexte de dyade collaborant à l'écriture d'un texte commun dans le cadre de l'acquisition d'une L2 (Apothéloz, 2005).

De même, en étudiant le rôle des reformulations dans le cadre d'entretiens à propos de l'activité d'infirmières en milieu hospitalier, Buléa (2010) défend la même idée car cela permet de « rendre compte de phénomènes relevant de l'activité verbale effective » (Buléa, 2010, p. 239) et de prendre en considération des unités linguistiques inférieures au mot tout en ajoutant une dimension praxéologique : «[...] la prise en compte simultanée des versants sémantique et structurel, et ce à un niveau dépassant les unités lexicales ou les relations interphrastiques, permet de mettre en évidence un des rôles majeurs que jouent les reformulations dans le processus de l'interprétation de l'agir » (ibid., p. 251).

Le rôle primordial du marqueur de reformulation (Gülich \& Kotschi, 1983) est aussi interrogé car les corpus oraux montrent que leur présence est exceptionnelle (Norén, 1999 ; Rabatel, 2007).

La plupart des recherches en contexte didactique (Volteau, 2009 ; Roquelaure, 2014 ; Moussi, 2015) utilisent le modèle classificatoire de Gülich \& Kotschi (1987) ou celui de De Gaulmyn (1987). Le premier modèle distingue trois actes de reformulation : le rephrasage, la paraphrase et la correction alors que dans le second modèle, le rephrasage est remplacé par la répétition. Ces modèles permettent-ils de rendre suffisamment compte des fonctions didactiques des reformulations?

\subsection{Parler pour apprendre à écrire (et à lire), parler pour comprendre les procédures d'écriture}

L'importance des pratiques d'écriture auprès de jeunes enfants est mis en évidence par des recherches de plus en plus nombreuses, celles-ci ayant été initiées par le courant des invented spelling (Chomsky, 1971 ; Read, 1971 ; Ferreiro \& Gómez Palacio, 1988). Certains travaux prennent en compte la dimension langagière au début de l'école primaire lors des pratiques d'écriture, d'une part pour apprendre à écrire (et à lire) mais aussi afin de comprendre les procédures d'écriture des élèves à travers les «commentaires métagraphiques » (Jaffré, 1995). Quelques travaux portant sur les reformulations en contexte scolaire d'écriture collaborative utilisent le modèle de Marty (1991).

\subsubsection{La dimension langagière des pratiques d'écriture au $C P$}

S'agissant du CP, soit pour justifier la pratique de l'écriture dès le début d'année (GarciaDebanc, 2003), soit dans le cadre de l' « atelier dirigé d'écriture » centré sur l'agir enseignant 
et les gestes professionnels (Bucheton \& Soulé, 2009), soit dans le cadre de l'analyse des interactions verbales en contexte de production écrite (Mauroux, David \& Garcia-Debanc, 2015), soit dans le cadre de l'analyse des écrits des élèves, des gestes d'étude et de la médiation enseignante (Champagne-Vergez \& Lubbers, 2019), les conclusions sont identiques : elles revendiquent l'interaction du lire-écrire-parler.

Les travaux de Bucheton et Soulé (2009) correspondent à notre contexte d'étude qui est celui de l'atelier de production d'écrits avec présence constante de l'adulte au CP. Les auteurs affirment que le «système interactionnel en écho (...) qui constitue une des composantes langagières de l'atelier, permet (...) de raisonner pas à pas sur les difficultés ponctuelles à partir d'une sélection-orientation-simplification des tâches » (ibid., p.141), les jeux de reformulations autour de l'écrit à produire ou produit les caractérisant : "le maître, qui regarde les élèves, répète à l'envie tout ou partie du mot à écrire ; les élèves, qui écrivent, répètent eux aussi à plus ou moins haute voix les fragments de mots voire les décomposent en unités plus petites encore au moment où ils les tracent » (ibid., p.141). Nous présentons un exemple à partir d'un extrait du corpus « Yaourt », les échanges portant sur l'écriture du mot « bonjour » :

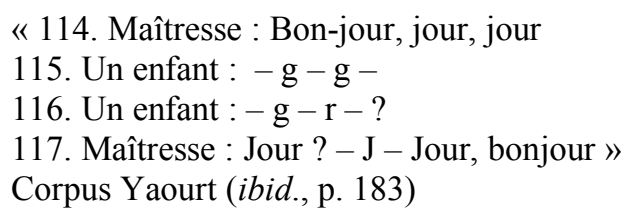

Les auteurs considèrent d'ailleurs que « la part langagière des gestes combinés du maître et des élèves » (ibid., p. 167) est déterminante pour la réussite d'un atelier. Leurs travaux appartiennent à un nouveau modèle qui semblent se dessiner depuis 2002, à savoir le modèle « centré sur l'écrivant dans la relation ajustée maitre/élève » (Bishop, 2019, p. 39). "La notion d'accompagnement dans l'acquisition du savoir écrire y occupe une place centrale » (ibid.), les gestes professionnels langagiers des enseignants jouant un rôle déterminant (Bucheton, 2014).

\subsubsection{Les commentaires métagraphiques: la mise en évidence des procédures d'écriture}

De plus en plus de chercheurs reconnaissent l'importance de croiser l'analyse des traces écrites du lecteur-scripteur avec les verbalisations de celui-ci (Jaffré, 2003 ; David, 2008 ; Morin, 2005 ; Mauroux \& Garcia-Debanc, 2019). Les travaux de Jaffré et David s'inscrivent dans le champ de la linguistique génétique : l'attention est portée sur le travail des jeunes scripteurs pour comprendre le processus d'acquisition de l'écriture, ainsi que sur l'objet écrit. Jaffré (2003) et David (2008) mettent en évidence l'intérêt des «commentaires métagraphiques » de jeunes enfants dans le cadre d'entretiens liés à leur production écrite, ceux-ci permettant de mieux comprendre les procédures d'acquisition de l'écriture.

L'analyse de ces "commentaires métagraphiques » est associée à l'analyse des écrits produits par les jeunes scripteurs de 5-7 ans, l'objectif étant de mieux comprendre les stratégies « intelligentes » mises en œuvre ainsi que les procédures déployées pour écrire. David (2008) relève notamment :

- la procédure phonographique qui consiste à établir une correspondance entre phonèmes et graphèmes, celle-ci comprenant les procédures phonogrammiques, analogiques, syllabiques et épellatives ;

- la procédure dite de « transport-copie » (Rieben, 1993) qui consiste à chercher un mot dans un écrit de référence pour le «transporter » avant de l'écrire. Cette procédure appartient à la procédure sémiographique qui inclut aussi l'écriture de mots mémorisés ; 
- la procédure morphographique qui consiste à écrire des marques dérivationnelles et/ou flexionnelles.

Ex. 1 (procédure phonographique) :

« Lorie (5,11 ans) - GS d'école maternelle

Récit de vie écrit en juin : Ce matin j'ai bu du coca-cola

EM pour semat (= ce matin)

Lol : je sais pas l'écrire alors j'ai cherché dans ma tête-j'ai dit [samatẽ] et j'ai

trouvé ces lettres là.

EM pour $c k c l$ (= coca-cola)

Lol : j'ai mis quatre lettres parce que ça fait quatre lettres dans [ko-ka-ko-la] et pi

[ka] je sais l'écrire c'est comme au début de Karine - c'est ma copine. "

Corpus David (2008, p. 170)»

Ex. 2 (procédure de « transport-copie ») :

« Jamila (5,6 ans) - GS d'école maternelle

Texte écrit en mai : david marion / piscine (= David et Marion vont à la piscine)

Ad : comment tu as fait pour écrire ces mots

Jam : et ben david et marion je sais déjà l'écrire - on l'a déjà mis ici (montre une affiche)

Ad : et pour piscine

Jam : je l'ai copié du livre de la classe. »

Corpus David (2008, p. 169)»

Ex. 3 (procédure morphographique) :

«...classe de CP, au début de novembre, lors de la découverte d'une phrase à analyser, dans une situation de dictée dialoguée : Dans la classe, huit enfants mangent à la cantine.

Ens : pourquoi on doit lire [mãj] et non [mãjã] ? À quoi ils servent le $N$ et le $T$ ?

Yéliz : c'est parce qu'y a plusieurs enfants qui mangent !»

Corpus David et Dappe (2011, p. 26)»

\subsubsection{Les reformulations en contexte scolaire d'écriture collaborative : "l'écrit dans l'oral ॥}

Marty (1991) étudie la genèse de l'écrit de trois élèves de CM2 qui écrivent un conte ensemble en se servant d'un traitement de texte sur ordinateur. Elle constate qu' " ils sont amenés à énoncer, de manière polyphonique, parfois conflictuelle, parfois plus constructive, de l'écrit dans l'oral » (Marty, 1991, p. 57). Elle présente sous forme de tableau la genèse de cet écrit pour chaque élève en s'inspirant du modèle syntaxique du Groupe Aixois de Recherche en Syntaxe (Blanche-Benveniste \& Jeanjean, 1987). Ce modèle est repris par Milian (2005) ainsi que Camps, Guasch, Milian et Ribas (2001) dans le cadre de l'étude de la reformulation orale en contexte d'écriture collaborative.

Milian (1999) analyse les interactions au sein de trilogues ou quadrilogues avec des collégiens catalans qui écrivent ensemble un texte explicatif en catalan sur le kaléidoscope : elle s'intéresse au rôle de la reformulation définie comme une "fonction rétroactive, de retour sur ce qui a été dit ou écrit, pendant le processus d'élaboration du discours » (Camps et al., 2001, p. 301). Pour l'auteure, la reformulation est un indice de l'activité métalinguistique mais aussi un moyen de construire des connaissances métalinguistiques (Milian, 2005), les reformulations étant souvent accompagnées d'énoncés rendant explicites une activité de réflexion sur la langue. 


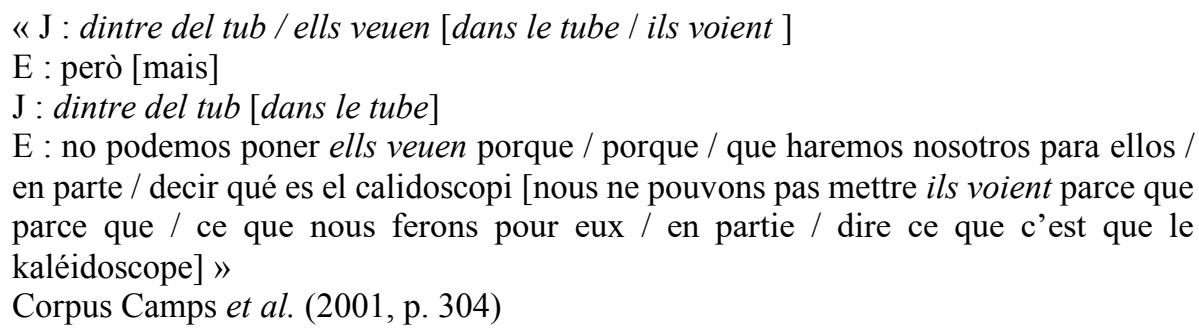

Dans cet extrait, les mots en italiques correspondent à de l' « écrit dans l'oral ». Le modèle de Marty (1991) permet « d'observer le processus de production d'un texte écrit à partir de l'oral et poursuivre, par les changements qui se produisent, les facteurs qui interviennent et qui ont une incidence sur la tâche envisagée et sur le contexte d'apprentissage » (ibid., pp. 300-301). Les auteurs soutiennent que « la reformulation constitue une sorte de brouillon oral qui permet de rendre objectif et de partager le texte que l'on est en train de produire » et qu'elle est un «important instrument d'apprentissage » (ibid., p. 308), notamment dans la construction du savoir sur le processus d'écriture et le développement des compétences métalinguistiques et métadiscursives (Milian, 2005).

\section{Méthodologie}

\subsection{Un corpus issu de la recherche Lire et Écrire au CP}

Nos données proviennent de la recherche Lire et Écrire au CP (IFÉ) intitulée «Étude de l'influence des choix didactiques des enseignants sur la qualité des apprentissages des élèves en lecture et en écriture entre 5 et 8 ans » (2012-2015). Les objectifs généraux de la recherche IFÉ (Goigoux, 2016) sont de dresser un état des pratiques effectives d'enseignement de la lecture et de l'écriture au $\mathrm{CP}$, de comparer les progrès des élèves entre les classes de l'échantillon (131 classes, 2500 élèves) et de tester des hypothèses sur ce qui peut faire la différence en matière d'efficacité des pratiques. Cette étude, qui permet d'observer les pratiques ordinaires, a donc une visée descriptive et une visée explicative (Crinon \& DiasChiaruttini, 2017).

Notre recherche (Owhadi, 2019) s'inscrit dans la continuité des travaux menés par le groupe Écriture (par ex. Dreyfus \& Brissaud, 2016) : les chercheurs de ce groupe s'attachent notamment à analyser les gestes professionnels du maitre lors des tâches d'écriture «afin d'arriver à une modélisation de la compétence professionnelle de l'enseignant » (ibid.).

Les résultats de l'étude IFÉ nous ont conduite à approfondir le rôle des reformulations orales considérées comme des phénomènes langagiers susceptibles d'expliquer les performances des élèves en écriture.

Notre corpus est constitué de onze séances d'ateliers de production d'écrits filmées et codées par les enquêteurs que nous avons retranscrites, celles-ci provenant de quatre classes contrastées en termes d'efficacité en écriture : la classe 61 (rang 8 / écriture), la classe 23 (rang 15 / écriture), la classe 106 (rang 98 / écriture) et la classe 68 (rang 118 / écriture). Le corpus étudié représente environ 6 heures 43 minutes. L'étude présente une dimension longitudinale, dans la mesure où, pour chacune des classes, ont été analysées au moins deux séances à des moments différents de l'année scolaire (novembre, mars et mai). 


\subsection{Le recueil des données}

\subsubsection{Un cheminement de pensée : les modalités d'analyse des reformulations}

Nous avons dans un premier temps utilisé le modèle classificatoire de Gülich et Kotschi (1987) affiné par De Gaulmyn (1987) : pour chaque reformulation, nous avons indiqué le type d'acte de reformulation (paraphrase, répétition ou correction) en précisant si c'est une auto-reformulation ou une hétéro-reformulation ainsi que le tour de parole où se situe l'énoncé-source (ES), celui-ci pouvant être séparé de l'énoncé reformulateur (ER) par des énoncés intercalés :

- la paraphrase: elle se caractérise par une relation sémantique entre l'ES et l'ER (équivalence sémantique de « faible » à « forte »).

601.M : non+ non+ non, + / il faut que ça aille avec + avec les cauchemars [ES]+ il faut que ça parle de la même chose [ER]+ il faut que les deux parties de la phrase elles parlent de la même chose, [ER]+/

(61, mai, séance 8)

- la répétition : les mêmes mots réapparaissent mais il peut y avoir abrègement, expansion ou réarrangement (De Gaulmyn, 1987). Pour cet acte de reformulation, l'équivalence sémantique est maximale entre l'énoncé-source et l'énoncé reformulateur.

308. M : (à une élève) hélicoptèrE [ES]+ hélicoptèrE, [ER]+/

(61, mai, séance 8)

- la correction : elle se caractérise par une relation sémantique de « contraste » (Gülich \& Kotschi, 1987) avec une modification d'un élément de l'énoncé-source considéré comme « fautif».

53.E1 : il y a des mamans i(ls) travaillent à la mairie, [ES]+/

54. M : il y a des mamans QUI travaillent à la mairie, [ER]+/

(61, mai, séance 8$)$

Ce modèle classificatoire ne permet pas suffisamment de rendre compte des fonctions des reformulations dans la situation spécifique d'accompagnement à l'écriture. Afin de montrer les insuffisances de ce modèle dans le cadre de notre contexte d'étude, nous présentons deux extraits de verbatim :

211. M : (à Au. en relisant sa phrase) je rêve que je suis un zèbre, $[\mathrm{ES}]+/$ (...) / (en

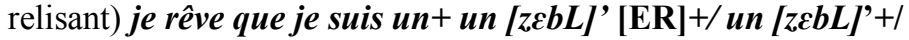

212. Hu. : un zèb-+ [b]+ [b]+ [b],+/

213. M : -bRe+ -bRe+-bRe,+/ qu'est'-ce que tu vas mettre, + /

214. Au. : $r-,+/$

215. $\mathrm{M}$ : et oui + donc on corrigera la prochaine fois,$+/$

(106, sem. 47, s. 21)

Au premier extrait, les jeux de reformulations autour de l'écrit produit montrent que celui-ci est repris-modifié par l'enseignant et les élèves en reprenant parfois une syllabe (bre) ou un phonème $[\mathrm{b}]$. Les modalités vocale/verbale semblent aussi intéressantes à prendre en considération, notamment la répétition questionnante et l'accentuation expressive 
(Guimbretière, 2014) : la maitresse utilise la reprise dubitative (Hudelot, 1997) à deux reprises (211 : «je rêve que je suis un zèble? un zèble ?) en accentuant sur le [1] afin qu'Audrey prenne conscience de son erreur. Elle répète à trois reprises (bre) avec accentuation sur le [r] afin d'inciter l'élève à utiliser une procédure phonographique (David,2008), l'effet de ces jeux de reformulations permettant à Audrey de comprendre son erreur $(214: \ll r »)$.

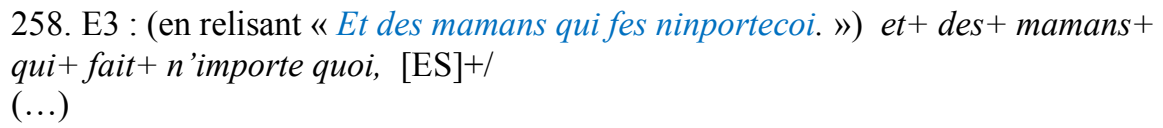

261. M : alors n'importe quoi ça s'écrit n'importe++++ quoi [ER],+/ écris-le moi correctement, $+/$ tout ça c'est + tout ça c'est la même phrase, + / oui' $+/$

262. E3 : non, +1

263. $\mathrm{M}:$ non'+/

264. E3: si,+/

265. M : si,+/ alors pourquoi est'-ce qu'il y a une majuscule ici,+++ (montre le mot « et ») / si c'est la même phrase+++ i(l) faut pas qu'i(l) y ait de majuscule+ en plein milieu, $+/ \mathrm{t}(\mathrm{u})$ es d'accord' $+/$ (en pointant successivement sur les mots écrits à corriger)

tu m'écris font+ n'importe++ quoi+ correctement, $[\mathrm{ER}]+$ /

266. E : n'importe quoi+ $\mathrm{i}(\mathrm{l})$ faut mettre un espace, + /

267. M : (geste de démarcation avec les mains) n'importe++++ quoi [ER],+/

268. E3 : i(l) faut mettre un espace, + +

(61, sem. 21, s. 8$)$

Dans ce deuxième extrait, nous avons la trace de l'écrit de l'élève E3 («Et des mamans qui fes ninportecoi. ») : les jeux de reformulations vont porter sur la procédure extragraphique (Catach, 1980/2003) consistant à segmenter des mots graphiques, le maitre essayant de faire comprendre à l'élève que «n'importe » et « quoi» sont deux mots distincts. La première reformulation du maitre utilise une pause exagérée entre «n'importe» et «quoi», « n'importe quoi » étant mis à distance (261: «alors n'importe quoi ça s'écrit n'importe quoi »). Il en va de même pour la deuxième reformulation à la différence que le maitre ajoute un geste de pointage sur les mots concernés (265: "tu m'écris font n'importe quoi correctement»). Un élève reformule ensuite avec de l'« écrit dans l'oral » distancé : « n'importe quoi il faut mettre un espace » (266). Le maitre reformule ensuite avec un geste de démarcation avec les mains et une pause exagérée entre «n'importe » et « quoi » (267). Cet extrait permet aussi de montrer l'intérêt de prendre en compte les modalités vocale/verbale avec la pause, les modalités gestuelle/verbale avec le déictique et l'illustratif selon la typologie de la gestualité co-verbale de Mc Neill (1992/2005). Il nous semble aussi intéressant de prendre en considération les reformulations avec de l' "écrit dans l'oral » distancé qui ont une dimension "méta » (le maitre et les élèves parlent du langage écrit). Enfin, les modalités oral/écrit (Cadet, 2014) mettent en exergue l'acte de lecture (l'écrit produit relu à voix haute au T258) ainsi que l'acte graphique : nous les avons nommées « modalités oralo-graphique » en empruntant « oralo-graphique » à Bouchard (1998).

Ainsi, afin de compléter le modèle classificatoire des actes de reformulation dans une perspective didactique, nous affinons notre analyse par la prise en compte de la multimodalité (Rabatel, 2010b, Cadet, 2014) ainsi que l'« écrit dans l'oral » distancé. Nous proposons une typologie des reformulations qui traitent de l'écrit à produire ou produit. Notre typologie précise les formes et les fonctions didactiques des reformulations dans la situation spécifique d'accompagnement à l'écriture sachant que nous intégrons dans notre analyse des reformulations des unités inférieures au mot (lettre, syllabe et phonème) car ces reformulations sont importantes dans notre contexte d'étude. 
Le tableau suivant permet de rendre compte des différents types de configurations de reformulations de l' « écrit dans l'oral », le choix des modalités étant didactiquement orienté :

\begin{tabular}{|c|c|c|c|}
\hline \multirow{4}{*}{$\begin{array}{l}\text { Modalités } \\
\text { vocale/verbale }\end{array}$} & RQ & $\begin{array}{l}\text { Répétition } \\
\text { Questionnante }\end{array}$ & $\begin{array}{l}\text { Répétitions avec intonation } \\
\text { montante en fin d'énoncé }\end{array}$ \\
\hline & $\overline{\mathrm{AE}}$ & $\begin{array}{l}\text { Accentuation } \\
\text { Expressive }\end{array}$ & $\begin{array}{l}\text { Reformulations avec accentuation } \\
\text { expressive sur un phonème (p) ou } \\
\text { une syllabe (s) }\end{array}$ \\
\hline & $\bar{P}$ & Pause & $\begin{array}{l}\text { Reformulations avec pauses } \\
\text { exagérées entre les phonèmes (p), } \\
\text { les syllabes (s) ou les mots }(\mathrm{m}) \text { ou } \\
\text { absence de pause entre les mots }(\varnothing)\end{array}$ \\
\hline & $\mathrm{D}$ & Déictique & Reformulations avec pointage \\
\hline $\begin{array}{l}\text { Modalités } \\
\text { gestuelle/ } \\
\text { verbale }\end{array}$ & I & Illustratif & $\begin{array}{l}\text { Reformulations avec mouvement } \\
\text { des mains et des bras sur un } \\
\text { phonème }(\mathrm{p}) \text {, entre les syllabes }(\mathrm{s}) \text {, } \\
\text { entre les mots }(\mathrm{m}) \text { ou à la fin d'un } \\
\text { énoncé }(\rightarrow)\end{array}$ \\
\hline $\begin{array}{l}\text { Modalités } \\
\text { oralo- } \\
\text { graphique }\end{array}$ & $\mathrm{AG}$ & Acte Graphique & $\begin{array}{l}\text { Reformulations associées à l'acte } \\
\text { graphique }\end{array}$ \\
\hline Modalité & $\overline{\mathrm{LHV}}$ & $\begin{array}{l}\text { Lecture à Haute } \\
\text { Voix } \\
\end{array}$ & $\begin{array}{l}\text { Reformulations associées à l'acte de } \\
\text { lecture }\end{array}$ \\
\hline «imension & EOD & $\begin{array}{l}\text { «Écrit dans } \\
\text { l'Oral » Distancé }\end{array}$ & $\begin{array}{l}\text { Reformulations avec de l'« écrit } \\
\text { dans l'oral » distancé }\end{array}$ \\
\hline
\end{tabular}

Tableau 1 : configurations de reformulations de l'«écrit dans l'oral » regroupées par modalité ou combinaison de modalités.

Ainsi, dans le cadre des modalités d'analyse des reformulations, nous avons ajouté les informations suivantes :

- lorsque la reformulation contient de l'« écrit dans l'oral», nous avons mentionné « écrit dans l'oral » entre parenthèses ;

- la description de la reformulation de l'« écrit dans l'oral » est indiquée en italiques selon le codage suivant : $R Q$ (Répétition Questionnante) ; $A E p$ (Accentuation Expressive sur un ou plusieurs phonèmes); AEs (Accentuation Expressive sur une ou plusieurs syllabes); $P p$ (Pause entre les phonèmes) ; Ps (Pause entre les syllabes) ; Pm (Pause entre les mots) ; Pø (absence de Pause) ; $D$ (gestuelle de type «Déictique ») ; Ip (Illustratif portant sur un phonème) ; Is (Illustratif portant sur des syllabes) ; Im (Illustratif portant sur un mot) ; $I \rightarrow$ (Illustratif à la fin d'un énoncé); $A G$ (Acte Graphique correspondant à toute trace laissée sur un support graphique); $L H V$ (Lecture à Haute Voix) ; EOD («Écrit dans l'Oral » Distancé). Le surlignage en bleu correspond à ce qui relève de la prosodie alors que celui en rouge correspond à la gestualité. Le surlignage en jaune correspond à la dimension oralo-graphique, le surlignage en orange correspondant à l'acte graphique qui relève à la fois de l'oralographique et de la gestualité. Enfin, le surlignage en vert correspond à de l' « écrit dans l'oral » distancé. Cette analyse fine des reformulations a permis de procéder à une quantification de celles-ci. 


\subsubsection{Méthode de transcription}

Les conventions de transcription du corpus s'inspirent de celles élaborées par Vion (1992) car elles prennent en compte les phénomènes de prosodie, qui sont des éléments déterminants d'analyse des reformulations. Chaque tour de parole est numéroté et tous les énoncés sont retranscrits intégralement en tenant compte de la fin d'un énoncé (barre oblique), des chevauchements de parole (soulignement des paroles perçues comme simultanées), du non verbal (description mise entre parenthèses) et de la prosodie (signe « + » indiquant une pause d'une seconde ; capitales d'imprimerie pour les unités linguistiques accentuées ; apostrophe pour l'intonation montante et virgule pour l'intonation descendante ; signe «: " pour l'allongement de la syllabe ou du phonème qui précède). Une interprétation orthographique est adoptée : les lettres correspondant à des sons non réalisés sont indiquées entre parenthèses. Les mots inaudibles d'une ou plusieurs syllabes sont indiqués par le signe "X séquences où la transcription est incertaine sont mises entre crochets. Les parties en italiques à l'intérieur des reformulations correspondent à de l' " écrit dans l'oral », les reformulations figurant en caractère gras. Les parties grisées correspondent à des échanges hors atelier d'écriture que nous avons décomptés. Les contraintes liées à la recherche Lire et Écrire au $C P$ ne nous ont pas permis de retranscrire les écrits produits par les élèves sauf dans la classe 61 : les écrits produits par l'élève figurent en italique bleu et ceux du maitre figurent en italique violet dans les parties entre parenthèses.

Les verbatims se présentent sous la forme d'un tableau à deux colonnes. À gauche, figure l'enchainement des tours de parole numérotés et transcrits alors que la colonne de droite est consacrée à l'analyse des reformulations (en caractère gras dans la colonne de gauche), celle des élèves étant soulignée.

\section{Quelques résultats}

Nous ne présentons que les résultats significatifs en lien avec notre typologie des reformulations de l' " écrit dans l'oral » dans le cadre de cette contribution compte tenu des contraintes de cet écrit.

\subsection{Analyses quantitatives}

\subsubsection{Les reformulations avec de l'"écrit dans l'oral " distancé comme indice de réflexivité de la part des élèves}

Du côté des élèves, voici le tableau avec le pourcentage de modalités des reformulations de l'« écrit dans l'oral » par rapport au nombre total de reformulations des élèves :

\begin{tabular}{|c|c|c|c|c|}
\hline & 61 & 23 & 106 & 68 \\
\hline Modalités vocale / verbale & $23 / 445$ & $9 / 219$ & $3 / 178$ & $3 / 151$ \\
& $5,16 \%$ & $4,1 \%$ & $1,68 \%$ & $1,98 \%$ \\
\hline Modalités & $18 / 445$ & $9 / 219$ & $3 / 178$ & $3 / 151$ \\
gestuelle / verbale & $4,0 \%$ & $4,1 \%$ & $1,68 \%$ & $1,98 \%$ \\
\hline Modalité oralo-graphique & $60 / 445$ & $16 / 219$ & $23 / 178$ & $13 / 151$ \\
& $13,48 \%$ & $7,3 \%$ & $12,92 \%$ & $8,6 \%$ \\
\hline Modalité verbale à dimension « méta » & $142 / 445$ & $48 / 219$ & $28 / 178$ & $12 / 151$ \\
& $31,91 \%$ & $21,91 \%$ & $15,57 \%$ & $7,94 \%$ \\
\hline
\end{tabular}

Tableau 2 : pourcentage de modalités des reformulations de l' "écrit dans l'oral 》 des élèves / nombre total de reformulations des élèves. 
On constate que plus la classe est efficace en écriture, plus les modalités vocale/verbale et gestuelle/verbale sont importantes dans les reformulations de l'«écrit dans l'oral» des élèves. Il en est de même pour la modalité verbale à dimension " méta », autrement dit aux reformulations avec de l' « écrit dans l'oral » distancé des élèves, l'écart étant assez important entre la classe la plus efficace $(31,91 \%$ dans la classe 61$)$ et la classe la moins efficace en écriture (7,94\% dans la classe 68$)$. On constate que les pourcentages de la modalité verbale à dimension «méta» des reformulations de l'«écrit dans l'oral» des élèves sont significativement différents dans les 4 classes (khi-2 $=34,18$; p ou seuil de significativité $<0,05)$. Cependant, les pourcentages des modalités vocale/verbale des reformulations de l'« écrit dans l'oral » des élèves ne sont pas significativement différents (khi-2 = 5,6) ainsi que ceux des modalités gestuelle/verbale (khi-2 =3,35).

\subsubsection{Les reformulations de l'"écrit dans l'oral » avec modalités gestuelle/verbale comme indice d'expertise langagière}

Si l'on s'intéresse maintenant aux reformulations de l' « écrit dans l'oral » du maitre ou de la maitresse, on se rend compte que plus la classe est efficace en écriture, plus l'enseignant utilise les modalités gestuelle/verbale comme le montre le tableau suivant :

\begin{tabular}{|c|c|c|c|c|}
\hline & 61 & 23 & 106 & 68 \\
\hline Modalités vocale / verbale & $139 / 1070$ & $155 / 386$ & $91 / 464$ & $114 / 588$ \\
& $12,99 \%$ & $40,15 \%$ & $19,61 \%$ & $19,38 \%$ \\
\hline Modalités & $241 / 1070$ & $80 / 386$ & $66 / 464$ & $52 / 588$ \\
gestuelle/ verbale & $22,52 \%$ & $20,72 \%$ & $14,22 \%$ & $8,84 \%$ \\
\hline Modalité oralo-graphique & $127 / 1070$ & $37 / 386$ & $67 / 464$ & $71 / 588$ \\
& $11,86 \%$ & $9,58 \%$ & $14,43 \%$ & $12,07 \%$ \\
\hline Modalité verbale à dimension & $363 / 1070$ & $151 / 386$ & $173 / 464$ & $191 / 588$ \\
« méta » & $33,92 \%$ & $39,11 \%$ & $37,28 \%$ & $32,48 \%$ \\
\hline
\end{tabular}

Tableau 3 : pourcentage de modalités des reformulations de l'« écrit dans l'oral » de l'enseignant / nombre total de reformulations de l'enseignant.

L'écart est deux fois et demi supérieur entre la classe 61 (22,52\%) et la classe 68 (8,84\%) si l'on prend en compte le nombre total de reformulations. On peut constater que les pourcentages de modalités gestuelle/verbale des reformulations de l'écrit dans l'oral sont significativement différents (khi-2 $=25,19)$.

\subsection{Une typologie qui permet d'établir un lien avec les procédures d'acquisition de l'écriture}

Notre typologie des reformulations de l'« écrit dans l'oral» permet de rendre compte des fonctions didactiques de celles-ci en lien avec les procédures d'acquisition de l'écriture (David, 2008). Nous avons ajouté la procédure extragraphique en empruntant le vocable « extragraphique » à Catach (1980/2003).

Le tableau suivant permet de faire une synthèse des fonctions à dominante didactique des reformulations de l' « écrit dans l'oral » de l'enseignant notamment lorsqu'il aide les élèves à produire de l'écrit. Il permet de percevoir les différentes configurations « typiques » pour chaque fonction didactique : 


\begin{tabular}{|l|l|}
\hline Procédure phonographique \\
\hline Phonogrammique \\
\hline $\begin{array}{l}\text { En accentuant sur un phonème } \\
\text { ou/et une syllabe }\end{array}$ & AEs / AEs-LHV / AEs-D / AEp / AEs-AG-EOD \\
\hline $\begin{array}{l}\text { En segmentant le mot en } \\
\text { syllabes }\end{array}$ & Ps / Ps-Is / Ps-Is-Im-EOD \\
\hline $\begin{array}{l}\text { En segmentant le mot en } \\
\text { phonèmes }\end{array}$ & AEp-Pp \\
\hline $\begin{array}{l}\text { En accentuant sur un phonème } \\
\text { ou/et une syllabe et en } \\
\text { segmentant le mot en syllabes }\end{array}$ & $\begin{array}{l}\text { AEp-Ps / AEp-AEs-Ps-D / AEp-AEs-Ps-EOD / } \\
\text { AEs-Ps-Is / AEs-Ps }\end{array}$ \\
\hline $\begin{array}{l}\text { Avec un geste illustratif associé } \\
\text { à un phonème pour en faciliter } \\
\text { la mémorisation }\end{array}$ & AEp-Ip / AEp-Ip-EOD \\
\hline Analogique \\
\hline $\begin{array}{l}\text { Avec de l'« écrit dans l'oral » } \\
\text { distancé }\end{array}$ & EOD / D-EOD / AEs-Is-EOD \\
\hline Procédure logographique \\
\hline Transport-copie & AEs - D -EOD / D-EOD \\
\hline Procédure morphographique \\
\hline Accord en nombre des noms & EOD / D-LHV \\
\hline Morphogramme lexical D & D-EOD / AEs-EOD \\
\hline Procédure extragraphique \\
\hline $\begin{array}{l}\text { Segmenter l'écrit avec des mots } \\
\text { graphiques }\end{array}$ & $\begin{array}{l}\text { Pm-EOD / Pm-D-EOD / Pm-Im / Pø-LHV-EOD / D- } \\
\text { LHV / AG-EOD }\end{array}$ \\
\hline
\end{tabular}

Tableau 4 : quelques fonctions didactiques des reformulations de l' "écrit dans l'oral 》 de l'enseignant en lien avec les procédures d'acquisition de l'écriture

Afin de mieux comprendre la lecture de ce tableau, nous prenons l'exemple de la procédure logographique: l'enseignant incite parfois l'élève à avoir recours à une procédure logographique par le «transport-copie » (Rieben, 2003) de mots disponibles dans la classe (affichages, carnet de mots, mot écrit au tableau, etc.). Les reformulations de ce type se caractérisent souvent par un pointage sur le mot à recopier avec de l'«écrit dans l'oral » distancé (D-EOD) avec parfois une accentuation expressive sur les mots monosyllabiques à recopier (AEs) :

27.M : (...) je vais dire je rêve que JE+ (en posant une main sur la poitrine) SUIS, $[\mathrm{ES}]+/$

(...)

59.M : (...) ALORS on a dit je rêve+ on l'a écrit au tableau (pointage du doigt sur "rêve») que (pointage du doigt sur «que») $\boldsymbol{J E}+$ on sait l'écrire+ mot du petit carnet et tu nous as dit SUIS, [ER]+/

(106, sem. 47, s. 9)

Elles permettent aussi d'aider l'élève à s'auto-corriger en ayant recours aux affichages de la classe comme au tour de parole 233 de l'exemple suivant, les deux énoncés reformulateurs ayant la configuration Déictique - «Écrit dans l'oral » distancé (D/EOD) :

233. M : (...) des [ES]+ des + des+/ attention,+ (en montrant le «e» de « de ») / ça en français ça chante $[\mathrm{e}],+/$ des,$+/$ comment' on écrit des + regarde, (pointe un affichage) $[\mathbf{E R}] /$ des + c'est écrit en haut, $[\mathbf{E R}]+(\mathrm{Ra}$. ajoute un $\mathrm{s}-)$ / $(61$, sem. 21, s. 8$)$ 


\section{Discussion}

Nous avons pu constater les limites des typologies utilisées dans des travaux précédents en contexte d'atelier de production d'écrits au CP : en effet, le "système interactionnel en écho » (Bucheton \& Soulé, 2009) qui le caractérise est constitué de nombreuses répétitions de lettres, de phonèmes et de syllabes permettant de décomposer le mot afin d'aider l'élève à produire de l'écrit. Ce constat nous a amenée à les intégrer comme unités d'analyse, d'où notre définition de la reformulation : toute unité linguistique produite dans l'interaction orale à partir d'une unité linguistique antérieure, à visée didactique. Cette définition est proche de celle d'Apothéloz (2007, p. 145) mais nous prenons en compte l'aspect sémantique et praxéologique dans une perspective didactique.

Compte tenu de ces limites, nous avons élaboré une typologie afin d'analyser les (jeux de) reformulations lors de phases où l'écrit à produire ou produit est discuté au sein des interactions, en prenant en compte le modèle de Marty (1991). Notre typologie élargit considérablement celles utilisées dans des travaux précédents en incluant les modalités gestuelles et vocales, les modalités oral/écrit (Cadet, 2014) qui permettent de prendre en compte l'acte graphique et l'acte de lecture (lecture à haute voix) et la modalité verbale à dimension «méta » rendant compte des reformulations où l'enseignant/l'élève parle de l'écrit.

En ce qui concerne cette dernière modalité, comme les travaux sur les entretiens métagraphiques (par ex. David, 2008) montrent l'intérêt de prendre en compte les verbalisations autour de l'écrit notamment du côté des élèves, nous avons considéré certaines reformulations avec de l'« écrit dans l'oral » distancé comme des paraphrases alors que l'on pourrait affirmer que ce sont plutôt des gloses :

268. M : (en relisant «Riana fai les toil de mère. ») riana+ fait+ l'étoile+ de mer, $[\mathrm{ES}]+/$ alors tss + tss + (avec geste de pointage sur " toil de mère ») ce qu'elle fait c'est une+ ce qu'elle fait c'est une+ [twal] de mère ou une Étoile de mer' [ER]/ (61, sem. 12, s. 4)

Cette recherche vient compléter les travaux sur l'étude des reformulations en contexte didactique, d'une part en élargissant l'empan de l'énoncé et en prenant ses distances par rapport à la définition classique de la reformulation orale, d'autre part en intégrant la multimodalité (Rabatel, 2010b; Cadet, 2014) et la dimension " méta », ainsi que les fonctions didactiques des reformulations spécifiques à l'enseignement/apprentissage de l'écriture au CP.

Notre typologie permet d'établir un lien avec les fonctions didactiques des reformulations à partir des procédures qui aident l'élève à produire de l'écrit, les critères d'analyse permettant de dépasser les dimensions langagières et linguistiques en prenant en compte la dimension didactique.

Cependant, cette typologie très détaillée rend le codage et le comptage difficiles et complexes. En outre, le fait d'avoir proposé une définition de la reformulation la plus extensive possible, ceci afin d'analyser l'ensemble des modalités de reformulation comme composante significative du travail verbal de la classe, a renforcé la difficulté à distinguer ce qui relève de la conversation ordinaire de ce qui relève de la didactique du français et donne parfois l'impression vertigineuse que tout est reformulation. De surcroit, notre approche dialogique qui prend en compte les « reformulations différées » (Gülich \& Kotschi, 1987) pose de redoutables problèmes d'analyse, l'énoncé-source étant parfois difficile à repérer.

Néanmoins, cela nous a permis de faire de la reformulation un " analyseur de l'activité enseignante » (Marin, 2011) voire de l'activité didactique et d'obtenir quelques résultats intéressants en procédant aux comptages des reformulations de l' ' écrit dans l'oral» : en comparant les reformulations de l'« écrit dans l'oral » du côté des élèves, nous avons pu 
mettre en évidence qu'il y a davantage de reformulations de l' « écrit dans l'oral » distancé, autrement dit « avec extraction de signes » (Bonnet \& Tamine-Gardes, 1984) dans les classes les plus efficaces en écriture. On peut ainsi faire l'hypothèse que la dimension réflexive est plus importante du côté des élèves dans les classes les plus efficaces en écriture, ceux-ci développant davantage de compétences métascripturales puisqu'ils parlent du langage écrit de manière plus importante.

Les analyses quantitatives montrent aussi que les enseignants des classes les plus efficaces en écriture utilisent davantage de reformulations de l'« écrit dans l'oral » avec modalités gestuelle/verbale. Ces résultats vont peut-être dans le même sens que les travaux de Jorro sur la corporéité de l'activité enseignante (Jorro, 2004 ; Jorro \& Crocé-Spinelli, 2010) qui montrent les difficultés des enseignants débutants à user d'un " corps parlant ", celui-ci semblant faire la différence en situation didactique (Jorro \& Crocé-Spinelli, 2010). Il semble cependant difficile de mesurer l'impact de la gestualité dans les interactions didactiques.

Nos résultats sont cependant à interpréter avec prudence : il semble difficile de généraliser ceux-ci au-delà de notre petit échantillon de quatre classes, d'autant plus que l'action est située donc dépendante du contexte. Ces éléments d'observation nécessiteraient d'être confirmés sur un échantillon plus large.

\section{Pour conclure}

Nous avons montré les limites des théories de référence sur la reformulation dans notre contexte d'étude : nous avons conçu une typologie des reformulations autour de l'écrit à produire ou produit qui permet de présenter les fonctions didactiques en lien avec les procédures d'écriture (David, 2008). Nous mettons en évidence l'intérêt de prendre en compte des unités linguistiques inférieures au mot, la multimodalité (Rabatel, 2010b ; Cadet, 2014) ainsi que la dimension « méta » dans notre contexte d'étude. En outre, l'analyse à grain fin des reformulations orales de notre corpus nous a permis de repérer des «gestes-témoins» (Soulé, 2008) autrement dit des gestes didactiques révélateurs de l'expertise enseignante qui pourraient être pointés en formation. Cependant, comment faire prendre conscience aux enseignants novices ou expérimentés de l'importance de ce phénomène langagier, partant de l'impact des gestes langagiers de reformulation sur les processus d'apprentissage?

\section{Références bibliographiques}

Apothéloz, D. (2005). Progression du texte dans les rédactions conversationnelles : les techniques de la reformulation dans la fabrication collaborative du texte. In R. Bouchard, L. Mondada (éds.), Les processus de la rédaction collaborative (pp. 165-199). Paris : L'Harmattan.

Apothéloz, D. (2007). Note sur l'activité de reformulation dans la conversation. Recherches linguistiques, 29, 145-162.

Bautier, É. (2001). Note de synthèse [Pratiques langagières et scolarisation]. Revue française de pédagogie, 137, 117-161.

Bishop, M.-F. (2019). L'enseignement de l'écriture à l'école primaire française de 1880 aux années 2000. In B. Kervyn, M. Dreyfus, C. Brissaud (dir.), L'écriture dès le début de l'école primaire (pp. 19-39). Bordeaux : Presses Universitaires de Bordeaux.

Blanche-Benveniste, C., Jeanjean, C. (1987). Le français parlé. Paris : Didier Érudition.

Bonnet, C., Tamine-Gardes, J. (1984). Quand l'enfant parle du langage : connaissance et conscience du langage chez l'enfant. Bruxelles : Pierre Mardaga éditeur.

Bouchard, R. (1998). L'interaction en classe comme polylogue praxéologique. Mélanges en hommage à Michel Dabène, ELLUG, Grenoble 3. Accessible en ligne

〈http ://www.inrp.fr/rencontres/seminaires /2004/ sem didac/ bouchard article 1.pdf $\rangle$. 
Brixhe, D., Spécogna, A. (1999). Actes de reformulation et progression du savoir. Pratiques, 103-104, 9-27.

Bucheton, D., Soulé, Y. (2009). L'atelier dirigé d'écriture au CP : une réponse à l'hétérogénéité des élèves. Paris : Delagrave.

Bucheton, D. (2014). Refonder l'enseignement de l'écriture. Paris : Retz.

Buléa, É. (2010). Signes, discours et interprétations de l'agir : le rôle des reformulations dans des entretiens portant sur le travail infirmier. In A. Rabatel (dir.), Les reformulations pluri-sémiotiques en contexte de formation (pp. 237-253). Besançon : Presses universitaires de Franche-Comté.

Cadet, L. (2014). Le corps de l'enseignant : sujet de la recherche, objet de la formation. In M. Tellier, L. Cadet (dir), Le corps et la voix de l'enseignant : théorie et pratique (pp.189-206). Paris : Éditions Maison des Langues.

Camps, A., Guasch, O., Milian, M., Ribas, T. (2001). L'écrit dans l'oral : le texte proposé. In M.-M. de Gaulmyn, R. Bouchard, A. Rabatel (éds.). Le processus rédactionnel : Écrire à plusieurs voix (pp. 293-310). Paris : L'Harmattan.

Catach, N. (1980/2003). L'orthographe française. Paris : Nathan.

Champagne-Vergez, M., Lubbers, M.-P. (2019). Écriture au cours d'une année dans une classe de CP : quelles modalités pour quels apprentissages ? In B. Kervyn, M. Dreyfus, C. Brissaud (dir.), L'écriture dès le début de l'école primaire (pp. 103-124). Bordeaux : Presses Universitaires de Bordeaux.

Chomsky, C. (1971). Write first, read later. Childhood Education, 47, 296-299.

Crinon, J., Dias-Chiaruttini, A. (2017). Interroger l'efficacité des pratiques d'enseignement de la lecture-écriture au Cours Préparatoire. Repères, 55, 7-20.

David, J. (2008). Les explications métagraphiques appliquées aux premières écritures enfantines, Pratiques, 139-140. Accessible en ligne 〈https://journals.openedition.org/pratiques/1230 $\rangle$.

David, J., Dappe, L. (2011). Interactions et morphologie grammaticale écrite à l'école primaire. Recherches, 54, 17-31.

De Gaulmyn, M.-M. (1987). Actes de reformulation et processus de reformulation. In P. Bange (éd.), L'analyse des interactions verbales. La Dame de Caluire. Une consultation (pp. 83-98). Berne : P. Lang.

Dreyfus, M., Brissaud, C. (2016). A.2.4. Questions de recherche : l'écriture. In R. Goigoux (dir.), Lire et écrire au CP, étude de l'influence des pratiques d'enseignement de la lecture et de l'écriture sur la qualité des premiers apprentissages (pp. 36-37). Accessible en ligne 〈http://ife.enslyon.fr/ife /recherche/lire-ecrire

Ferreiro, E., Gómez Palacio, M. (1988). Lire-écrire à l'école. Comment s'y apprennent-ils ? Analyse des perturbations dans les processus d'apprentissage de la lecture et de l'écriture. Lyon: CRDP.

Fuchs, C. (1982). La paraphrase. Paris : PUF.

Garcia-Debanc, C. (2003). Faire écrire les élèves dès le début du CP. In B. Germain, I. Le Guay, N. Robert (dir.), Le manuel de lecture au CP. Réflexions, analyses et critères de choix (pp. 67-77). Paris : Savoir Livre/CNDP.

Garcia-Debanc, C., Plane, S. (coord.). (2004). Comment enseigner l'oral à l'école primaire? Paris : Hatier.

Garcia-Debanc, C., Volteau, S. (2007). Formes linguistiques et fonctions des reformulations dans les interactions scolaires. Recherches linguistiques, 29, 309-340.

Garcia-Debanc, C. (2008). Quelle place des sciences du langage dans la didactique du français en 2008 ? In J. Durand, B. Habert, B. Laks (éds.), Congrès Mondial de Linguistique Française, 9-12 juillet 2008, Paris, France (p. 605). Les Ulis : EDP Sciences.

Goigoux, R. (2016). Rapport de recherche Lire et Écrire : étude de l'influence des pratiques d'enseignement de la lecture et de l'écriture sur la qualité des premiers apprentissages. Institut Français de l'Éducation, ENS et Université de Lyon.

Guimbretière, É. (2014). Le corps et la voix de l'enseignant : une mise en contexte théorique et pratique. In M. Tellier, L. Cadet (dir.), Le corps et la voix de l'enseignant : théorie et pratique (pp. 15-27). Paris : Éditions Maison des Langues.

Gülich, É., Kotschi, T. (1983). Les marqueurs de la reformulation paraphrastique. Cahiers de linguistique française, 5 , 305-351.

Gülich, É., Kotschi, T. (1987). Les actes de reformulations dans la consultation la dame de Caluire. In P. Bange (éd.), L'analyse des interactions verbales. La dame de Caluire : une consultation (pp. 15-81). Berne : Peter Lang. 
Hudelot, C. (1997). Modalités d'intervention de l'adulte dans la gestion d'un petit groupe d'enfants de moyenne section en situation de description d'image. Cahiers du Calap, 14, 123-155.

Jaffré, J.-P. (1995). Les explications métagraphiques. Leur rôle en recherche et en didactique. In R. Bouchard, J.-C. Meyer (coll.), Les métalangages de la classe de français (pp. 137-138). Actes du 6ème colloque de Lyon, 20-23 septembre 1995. Lyon : DFLM.

Jaffré, J.-P. (2003). Les commentaires métagraphiques. Faits de langues, 22, 67-76.

Jaubert, M., Rebière, M. (2001). Pratiques de reformulation et construction de savoirs. ASTER, 33, 81110.

Jorro, A. (2004). Le corps parlant de l'enseignant. Entente, malentendus, négociations. Actes du $9^{\mathrm{e}}$ colloque de l'AIRDF, Québec, aout 2004. Accessible en ligne 〈http: //www.colloqueairdf.fse.ulaval.ca/fichier/Symposium Bucheton/Jorro.pdf).

Jorro, A., Crocé-Spinelli, H. (2010). Le développement de gestes professionnels en classe de français. Le cas de situations de lecture interprétative. Pratiques, 145/146, 125-140.

Marin, B. (2011). La reformulation en classe : un discours équivoque. In J.-Y. Rochex, J. Crinon (dir.), La construction des inégalités scolaires. Au cœur des pratiques et des dispositifs d'enseignement (pp. 77-88). Rennes : Presses Universitaires de Rennes.

Martinot, C. (2000). Étude comparative des processus de reformulation chez des enfants de 5 à 11 ans. Langages, 140, 92-123.

Marty, N. (1991). Genèse de l'écrit et activités métalinguistiques dans le dialogue de jeunes scripteurs. Etudes de Linguistique Appliquée, 81, 57-70.

Mauroux, F., David, J., Garcia-Debanc, C. (2015). Analyse des actions et interactions didactiques en production écrite au Cours Préparatoire. Repères, 52, 121-141.

Mauroux, F., Garcia-Debanc, C. (2019). Étude longitudinale des effets des écritures approchées sur les compétences des apprentis-scripteurs au début de l'école primaire en France. In B. Kervyn, M. Dreyfus, C. Brissaud (dir.), L'écriture dès le début de l'école primaire (pp. 43-64). Bordeaux : Presses Universitaires de Bordeaux.

Mc Neill, D. (1992/2005). Gesture and thoughts. Chicago : The University of Chicago Press.

Milian, M. (1999). Interacció de contextos en el procés de composició escrita en grup. Capítol 10 : Les reformulacions (pp. 301-367) (Thèse doctorale). Universitat Autònoma de Barcelona.

Milian, M. (2005). Reformulation : a Means of Constructing Knowledge in Shared Writing. Educational Studies in Language and Literature, 5 (3), 335-351.

Morin, M.-F. (2005). Declared Knowledge of Beginning Writers. L1-Educational Studies in Language and Literature, 5 (3), 385-401.

Moussi, D. (2013). Intérêts et enjeux d'une révision interactive des textes au cycle 3 : le rôle de l'enseignant (Thèse de doctorat). Université Lille III.

Moussi, D. (2015). Re-formuler...Ou comment prendre en compte les réponses des élèves pour avancer dans l'interaction? Recherches, 62, 51-71.

Norén, C. (1999). Reformulation et conversation. De la sémantique du topos aux fonctions interactionnelles. Uppsala : Acta Universitatis Upsaliensis.

Owhadi, H. (2019). Les reformulations orales en contexte d'atelier de production d'écrits au Cours Préparatoire. Étude linguistique des jeux de reformulations (Thèse de Doctorat). Université Paul Valéry - Montpellier 3.

Peytard, J. (1993). D'une sémiotique de l'altération. Revue de sémio-linguistique des textes et discours, $8,137-169$.

Rabatel, A. (2007). Répétitions et reformulations dans l'Exode: coénonciation entre Dieu, ses représentants et le narrateur, Recherches linguistiques, 29, 75-96.

Rabatel, A. (dir.). (2010a). Les reformulations pluri-sémiotiques en contexte de formation. Besançon : Presses universitaires de Franche-Comté.

Rabatel, A. (2010b). Pour une approche intégrée des reformulations pluri-sémiotiques en contexte de formation : apprendre en reformulant et en resémiotisant documents iconiques, gestes et actions. In A. Rabatel (2010), Les reformulations pluri-sémiotiques en contexte de formation (pp. 7-24). Besançon : Presses universitaires de Franche-Comté.

Rabatel, A. (2017). Reformulation, anaphore résumante/conceptuelle et formule. Résumé de la conférence plénière au colloque «La reformulation: à la recherche d'une frontière », 8-9 juin 2017. Université d'Uppsala, Suède. Accessible en ligne 〈http: //www.moderna.uu.se/digitalAssets/635/c 635110-1_3-k_plenieres.pdf).

Read, C. (1971). Pre-school children's knowledge of english phonology. Harvard Educational Review, 41,1-34. 
Reuter, Y. (éd.). (2007/2010). Dictionnaire des concepts fondamentaux des didactiques. Bruxelles : De Boeck.

Rieben, L. (1993). Production écrite en situation de classe et acquisition de connaissances lexicales. In J.-P. Jaffré, L. Sprenger-Charolles, M. Fayol (dir.), Lecture-écriture : acquisition. Les Actes de la Villette (pp. 137-151). Paris : Nathan.

Rieben, L. (2003). Écritures inventées et apprentissage de la lecture et de l'orthographe. Faits de langues, 14, 27-36.

Roquelaure, M.-F. (2014). Reformulations dans l'enseignement supérieur : discours du professeur et prises de notes des étudiants (Thèse de doctorat). Université Toulouse II - Le Mirail.

Soulé, Y. (2008). Troisième étude de cas : débat philo ou débat littéraire ? In D. Bucheton, O. Dezutter (dir.), Le développement des gestes professionnels dans l'enseignement du français (pp. 53-58). Bruxelles : De Boeck.

Vion, R. (1992). La communication verbale. Analyse des Interactions. Paris : Hachette.

Volteau, S. (2009). Les reformulations orales dans des interactions didactiques au cycle 3 de l'école primaire : formes et fonctions des reformulations d'une enseignante expérimentée et d'une enseignante débutante dans une classe de CM2 (Thèse de doctorat). Université de Toulouse II Le Mirail. 